\title{
Phase behavior of symmetric rod-plate mixtures revisited: Biaxiality versus demixing
}

\author{
Szabolcs Varga, Amparo Galindo, and George Jackson \\ Department of Chemical Engineering and Chemical Technology, Imperial College of Science, \\ Technology and Medicine, Prince Consort Road, London SW7 2BY, United Kingdom
}

(Received 31 July 2002; accepted 16 September 2002)

\begin{abstract}
The phase behavior of symmetric binary rod-plate mixtures has been investigated by numerical minimization of a free energy functional derived by Parsons [J. D. Parsons, Phys. Rev. A 19, 1225 (1979)] and Lee [S. D. Lee, J. Chem. Phys. 87, 4972 (1987)]. Both rod and plate molecules are represented by hard cylinders, with aspect ratios chosen so that the molecular and pair excluded volumes are equal; in this way symmetric phase diagrams in composition are found. The subtle competition between the packing entropy and the entropy of mixing rules out the possibility of a uniaxial nematic-biaxial nematic phase transition and instead favors a demixing phase transition between a rod-rich and a plate-rich nematic phase. It is shown that the biaxial nematic phase is unstable relative to demixing even for symmetric mixtures of very long rod and very flat plates, where the Parsons-Lee theory becomes identical with the Onsager theory. The contradictory predictions obtained in recent studies regarding the stability of the biaxial nematic phase have been resolved by examining the lowest aspect ratio of the rods $\left(\kappa_{2}\right)$ where the Parsons-Lee and Onsager theories become equivalent. It turns out that neglecting the lower order terms in the excluded volumes (so-called end effects) leads to a favoring of the biaxial nematic phases. Only two types of phase transitions are observed in this work: isotropic-nematic phase coexistence and demixing transitions involving either two isotropic or two nematic phases. The stability of the nematic region on mixing is found to be very sensitive to the aspect ratios of the molecules: for moderate aspect ratios of the rods $\left(5<\kappa_{2}<10\right)$, a destabilization of the nematic phase is observed relative to the isotropic phase, while for $\kappa_{2}>10$ the opposite tendency is found. A demixing transition between rod-rich and plate-rich nematic phases takes place for all of the systems studied with a widening coexistence region for increasing aspect ratio. Isotropic-isotropic demixing is also observed for aspect ratios $\kappa_{2}>65.5$. For the larger values of $\kappa_{2}$ the regions of isotropic-isotropic and nematicnematic demixing broaden, while the isotropic-nematic coexistence is progressively suppressed. (C) 2002 American Institute of Physics. [DOI: 10.1063/1.1519536]
\end{abstract}

\section{INTRODUCTION}

The fine balance between the orientational entropy (characterized by the orientational ordering in the system), the translational entropy (characterized by the shapes of the molecules and the free volume accessible to them), and the mixing entropy (characterized by the well-known ideal expression which is a function of the logarithm of the mole fractions of each component) determines the phase behavior of mixtures of hard-core liquid crystal systems. The possibility of biaxial nematic phases in mixtures of prolate and oblate molecules has attracted a great deal of interest and controversy in recent years. There are a large number of studies in this area, and for the sake of brevity we point the reader to recent work in which the topic is reviewed in some detail. ${ }^{1-3}$

Our current paper forms part of a series in which we examine the phase behavior of mixtures of generic repulsive rodlike and platelike molecules. In our first paper ${ }^{1}$ we concentrated on mixtures which were symmetric at the level of the second virial theory (so that the like excluded volumes are equal and as a consequence the phase behavior of the two pure components is identical), and the particles were examined in the Onsager limit (infinite aspect ratios, where only the leading terms of the excluded volumes are retained). The effect of varying the unlike rod-plate excluded volumes on the phase behavior was examined in detail. It was shown that different conclusions can be reached about the stability of the biaxial phase depending on the approximation that is used to represent the excluded volumes. In the case of the so-called L2 approximation (second-order Legendre representation of the excluded volume) a second-order nematic-biaxialnematic phase transition was observed for small unlike excluded volumes while the biaxial phase was found to be unstable with regard to a nematic-nematic demixing transition (in some cases between two isotropic phases) for large unlike excluded volumes. In the case of a full numerical solution of the excluded volume the biaxial phase was found to be stable with respect to nematic-nematic demixing, a striking result which is in contrast with previous findings (see Ref. 1 and references therein). We return to this point in our current paper.

Asymmetric mixtures of rod- and plate-like particles have also been examined in order to make contact with recent experimental work on colloidal systems by van der Kooij and Lekkerkerker. ${ }^{4-8}$ In this case the volumes (and excluded volumes) of the rods and plates are different, which adds asymmetry to the phase behavior; in their work the 
"rods" have an aspect ratio of about 10, and the "plates" of about $1 / 15$ which means that the excluded volume of the plate is much larger than that of the rod. In these experimental studies a biaxial nematic phase was not observed, and instead the system exhibits large regions of nematic-nematic (and nematic-columnar) demixing. We have recently considered mixtures in which the volume of the plate is orders of magnitude larger than the volume of the $\operatorname{rod}^{2}{ }^{2}$ so that an equivalence can be made with experiment where the plates are colloidal particles while the rods play the role of a depleting agent. We used the Parsons-Lee scaling ${ }^{9,10}$ of the Onsager free energy in order to introduce the higher virial terms, and the lower order terms of the pair excluded volume (so-called end effects) were also included. This approach provides a more accurate representation of systems with smaller aspect ratios, as well as treating the fine balance between the like and unlike excluded volumes which gives rise to the demixing behavior. A combined analysis of the isotropic-nematic bifurcation transition and spinodal demixing was carried out to determine the geometrical requirements for the stabilization of a demixing transition involving two isotropic phases, and global phase diagrams were presented in terms of the molecular parameters. The stability analysis indicates that for certain aspect ratios, the isotropicnematic transition always pre-empts the demixing of the isotropic phase, irrespective of the diameters of the particles. When isotropic-isotropic demixing is found, there is an upper bound at large size ratios (Asakura and Oosawa limit), and a lower bound at small size ratios beyond which the system exhibits a miscible isotropic phase. We did not include an analysis of the biaxial phase in Ref. 2 as the focus was on the global isotropic-isotropic demixing and phase behavior at moderate pressures. The stability of the biaxial phase with respect to demixing in these asymmetric mixtures of rods and plates has recently been considered by Wensink et $a l^{3,11}$ They present a very rich and interesting phase behavior in such systems, including uniaxial nematic-biaxial nematic, and uniaxial nematic-uniaxial nematic demixing transitions for increasing asymmetry between the two components. In their work, however, although the Parsons-Lee approximation for the higher virial terms was used, only the leading term of the excluded volume was treated. As we shall see from the results presented in our current paper for the symmetric rod-plate mixture, this may give rise to some misleading conclusions about the relative stabilities of the biaxial and demixed states.

Here, we examine the phase behavior of symmetric mixtures of hard rods and plates using the numerical solution of the Parsons-Lee free energy functional incorporating the complete excluded volumes (end effects). We will show that the biaxial nematic phase turns out to be unstable relative to demixing even for a mixture of very long rods and very flat plates, where the Parsons-Lee theory becomes equivalent to the Onsager theory. This paper is organized as follows: The Parsons-Lee theory for symmetric binary mixtures is presented in the following section. Expressions are then given for the equilibrium orientational distribution functions, the uniaxial and biaxial order parameters, isotropic-nematic bifurcation analysis, and demixing transitions involving two isotropic (or nematic) phases. We also discuss the relation between the Onsager and Parsons-Lee theories, and an analytical expression is derived for the demixing transition between two isotropic phases in the Onsager approach. The molecular model considered in this work to represent the rod and platelike particles (the hard-cylinder model) is introduced at the end of the following section. The results of the demixing analysis, the isotropic-nematic bifurcation analysis, and the isotropic-nematic phase transition calculations for binary symmetric mixtures of hard cylinders are presented in Sec. III. Particular attention is paid to resolving the issue regarding the stability of the biaxial-nematic phase in rod-plate mixtures by means of Gibbs free energy calculations. Conclusions are given in Sec. IV.

\section{PARSONS-LEE THEORY}

One of the most successful theories of the phase behavior of nonspherical hard-body fluids, which is capable of accurately describing the isotropic-nematic phase transition for prolate or oblate particles, is due to Parsons ${ }^{9}$ and Lee. ${ }^{10}$ The theory provides a good representation of the transition properties, and the resulting equation of state reproduces the isotropic and nematic branches of the equation of state up to very high densities, where the more ordered smectic and columnar phases usually appear. Recent simulation and theoretical studies have shown that the density dependence of the pressure in fluids of hard-spherocylinders ${ }^{12}$ and uniaxial hard ellipsoids ${ }^{13}$ is accurately captured in the Parsons-Lee theory for a wide range of molecular geometries (aspect ratios). The theory can be considered as a scaling approach to Onsager's second virial theory, ${ }^{14}$ and differs only by a factor which depends on the shape of the particle and the density of the system; the two approaches become identical in the limit of very long or very flat particles. In the Parsons-Lee approach, in addition to the pair excluded volumes of the particles (which contain the molecular parameters), an expression for the residual free energy of the hard-sphere fluid is also required. The Carnahan-Starling free energy of the hardsphere fluid ${ }^{15}$ is typically used, and the Parsons-Lee theory reduces to this in the hard-sphere limit due to the inherent scaling to a hard-sphere reference. The simplest way to generalize the Parsons-Lee theory to binary mixtures is to map the binary system on to an effective one-component hardsphere system (van der Waals one-fluid theory ${ }^{16}$ ), and to use the excluded volumes and the residual Carnahan and Starling free energy expression of the effective pure hard-sphere fluid. The application of the one-fluid Parsons-Lee theory has proved successful in studies of ellipsoidal rod-plate mixtures ${ }^{17}$ and spherocylinder-cutsphere mixtures. ${ }^{18}$ Here, we use the same approximation because of its simplicity, but the elaboration of a Parsons-Lee approach based on the mapping onto a binary mixture of hard spheres of different diameter (two-fluid theory) may be necessary in the future.

In the one-fluid hard-sphere approximation of the Parsons-Lee theory, the Helmoltz free energy of the binary mixture can be written as a functional of the orientational distribution functions $\left(f_{i}, i=1,2\right)$ of the constituent molecules 


$$
\frac{\beta F}{N}=\sum_{i=1}^{2} x_{i}\left(\ln \rho-1+\ln \frac{v_{i}}{4 \pi}+\ln x_{i}+\sigma\left[f_{i}\right]\right)+\frac{\beta F_{\mathrm{ex}}^{\mathrm{hs}}}{N} \frac{\iint\left\{x_{1}^{2} v_{11}^{\mathrm{exc}} f_{1} f_{1}+2 x_{1} x_{2} v_{12}^{\mathrm{exc}} f_{1} f_{2}+x_{2}^{2} v_{22}^{\mathrm{exc}} f_{2} f_{2}\right\} d \underline{\omega}_{1} d \underline{\omega}_{2}}{8 v_{\mathrm{hs}}},
$$

where $\beta=1 / k T$ ( $T$ is the temperature and $k$ is Boltzmann's constant), $\rho=N / V$ is the number density, $x_{i}$ is the mole fraction, and $v_{i}$ the de Broglie volume, which takes into account the translational and rotational contributions to the kinetic energy of each component $i$. The orientational entropy term $\sigma\left[f_{i}\right]$ depends on the orientational distribution function $f_{i}$ of component $i$, so that

$$
\sigma\left[f_{i}\right]=\int f_{i}(\underline{\omega}) \ln \left(4 \pi f_{i}(\underline{\omega})\right) d \underline{\omega},
$$

where $\underline{\omega}$ is the orientational unit vector parallel to the symmetry axis of the particle; it is defined by a polar angle $(0$ $<\theta<\pi)$ and an azimuthal angle $(0<\varphi<2 \pi)$. The residual term of the free energy functional [the last term in Eq. (1)] takes into account the free energy gain due to molecular ordering through a minimization of the mole fractionweighted excluded volumes $v_{i j}^{\text {exc }}$. Note that, for simplicity, the orientational dependence of the excluded volumes $\left[v_{\mathrm{ij}}^{\text {exc }}\right.$ $=v_{i j}^{\operatorname{exc}}(\gamma)$, where $\left.\gamma=\arccos \left(\underline{\omega}_{i} \underline{\omega}_{j}\right)\right]$, and of the orientational distribution functions $\left(f_{i}=f_{i}(\underline{\omega})\right)$ have not been shown in Eq. (1). According to the original prescription of Parsons and Lee, ${ }^{9,10}$ the packing fraction of the system $\eta$, and that of reference hard-sphere fluid $\eta_{\mathrm{hs}}$ must be the same, which means that

$$
v_{\mathrm{hs}}=x_{1} v_{1}+x_{2} v_{2},
$$

where $v_{\text {hs }}$ is the volume of the hard sphere, and $v_{i}$ is the molecular volume of each of the components. The residual free energy of the hard-sphere fluid is represented by the Carnahan and Starling expression ${ }^{15}$ as

$$
\frac{\beta F_{\mathrm{ex}}^{\mathrm{hs}}}{N}=\frac{4 \eta-3 \eta^{2}}{(1-\eta)^{2}}
$$

Since we are only focusing on symmetric mixtures at this stage, the excluded volumes of the like interactions and the volumes of the species have to be identical, i.e., $v_{11}^{\mathrm{exc}}=v_{22}^{\mathrm{exc}}$ and $v_{\mathrm{hs}}=v_{1}=v_{2}$. In the appendix, we examine the necessary conditions for hard cylinder and hard spherocylinder systems to exhibit symmetric phase behavior. For the sake of generality, however, we do not specify the excluded volumes at this stage. The equilibrium orientational distribution functions are obtained by minimization of the free energy functional given in Eq. (1),

$$
\frac{\delta\left(\beta F / N+\lambda_{i}\left(1-\int f_{i}(\underline{\omega}) d \underline{\omega}\right)\right)}{\delta f_{i}(\underline{\omega})}=0,
$$

where the Lagrange multipliers $\lambda_{i}$ ensure the normalization conditions of the distribution functions $\left[\int f_{i}(\underline{\omega}) d \underline{\omega}=1, i\right.$ $=1,2]$. Substituting Eq. (1) into Eq. (5) results in two equations for the orientational distribution functions,

$$
\ln \left(4 \pi f_{1}(\underline{\omega})\right)=\lambda_{1}-\frac{\beta F_{\mathrm{ex}}^{\mathrm{hs}}}{N} \frac{\left(x_{1} \int v_{11}^{\mathrm{exc}}\left(\underline{\omega}_{2}\right) f_{1}\left(\underline{\omega}_{2}\right) d \underline{\omega}_{2}+x_{2} \int v_{12}^{\mathrm{exc}}\left(\underline{\omega}_{2}\right) f_{2}\left(\underline{\omega}_{2}\right) d \underline{\omega}_{2}\right)}{4 v_{\mathrm{hs}}}
$$

and

$$
\ln \left(4 \pi f_{2}(\underline{\omega})\right)=\lambda_{2}-\frac{\beta F_{\mathrm{ex}}^{\mathrm{hs}}}{N} \frac{\left(x_{2} \int v_{22}^{\mathrm{exc}}\left(\underline{\omega}_{\omega_{2}}\right) f_{2}\left(\underline{\omega}_{2}\right) d \underline{\omega}_{2}+x_{1} \int v_{12}^{\mathrm{exc}}\left(\underline{\omega}_{\omega_{2}}\right) f_{1}\left(\underline{\omega}_{2}\right) d \underline{\omega}_{2}\right)}{4 v_{\mathrm{hs}}} .
$$

Note that the conditions of symmetry have been used in the derivation of Eqs. (6) and (7). By integrating out the Lagrange multipliers two self-consistent equations for the distribution functions can be obtained after some manipulation,

$$
f_{1}(\underline{\omega})=\frac{\exp \left(-\frac{\beta F_{\mathrm{ex}}^{\mathrm{hs}}}{N} \frac{\left(x_{1} \int v_{11}^{\mathrm{exc}}\left(\underline{\omega}_{2}\right) f_{1}\left(\underline{\omega}_{2}\right) d \underline{\omega}_{2}+x_{2} \int v_{12}^{\mathrm{exc}}\left(\underline{\omega}_{2}\right) f_{2}\left(\underline{\omega}_{2}\right) d \underline{\omega}_{2}\right)}{4 v_{\mathrm{hs}}}\right)}{\int \exp \left(-\frac{\beta F_{\mathrm{ex}}^{\mathrm{hs}}}{N} \frac{\left(x_{1} \int v_{11}^{\mathrm{exc}}\left(\underline{\omega}_{1} \underline{\omega}_{2}\right) f_{1}\left(\underline{\omega}_{2}\right) d \underline{\omega}_{2}+x_{2} \int v_{12}^{\mathrm{exc}}\left(\underline{\omega}_{1} \underline{\omega}_{2}\right) f_{2}\left(\underline{\omega}_{2}\right) d \underline{\omega}_{2}\right)}{4 v_{\mathrm{hs}}}\right) d \underline{\omega}_{1}}
$$

and

$$
f_{2}(\underline{\omega})=\frac{\exp \left(-\frac{\beta F_{\mathrm{ex}}^{\mathrm{hs}}}{N} \frac{\left(x_{2} \int v_{22}^{\mathrm{exc}}\left(\underline{\omega}_{2}\right) \underline{\omega}_{2}\left(\underline{\omega}_{2}\right) d \underline{\omega}_{2}+x_{1} \int v_{12}^{\mathrm{exc}}\left(\underline{\omega}_{\omega_{2}}\right) f_{1}\left(\underline{\omega}_{2}\right) d \underline{\omega}_{2}\right)}{4 v_{\mathrm{hs}}}\right)}{\int \exp \left(-\frac{\beta F_{\mathrm{ex}}^{\mathrm{hs}}}{N} \frac{\left(x_{2} \int v_{22}^{\mathrm{exc}}\left(\underline{\omega}_{1} \underline{\omega}_{2}\right) f_{2}\left(\underline{\omega}_{2}\right) d \underline{\omega}_{2}+x_{1} \int v_{12}^{\mathrm{exc}}\left(\underline{\omega}_{1} \underline{\omega}_{2}\right) f_{1}\left(\underline{\omega}_{2}\right) d \underline{\omega}_{2}\right)}{4 v_{\mathrm{hs}}}\right) d \underline{\omega}_{1}} .
$$


This set of integral equations can be solved numerically without approximations. We do not present details of the numerical technique here, as these have been given in a previous paper. ${ }^{1}$ Once the Helmholtz free energy is obtained, the pressure $P$ and the chemical potentials $\mu_{i}$, can be determined using standard thermodynamic relations: $P$ $=-(\partial F / \partial V)_{N, T}$ and $\mu_{i}=\left(\partial F / \partial N_{i}\right)_{N_{j \neq i}, V, T}$. In practice it is more convenient to use dimensionless variables, so that a reduced pressure and chemical potential are defined as

$$
P^{*}=\beta P v_{\mathrm{hs}}=\eta^{2}\left(\frac{\partial \beta F / N}{\partial \eta}\right)
$$

and

$$
\begin{aligned}
\mu_{i}^{*}=\beta \mu_{i}= & \frac{\beta F}{N}-\sum_{j=1}^{2}\left(\frac{\partial \beta F / N}{\partial x_{j}}\right) x_{j} \\
& +\left(\frac{\partial \beta F / N}{\partial x_{i}}\right)+\left(\frac{\partial \beta F / N}{\partial \eta}\right) \eta .
\end{aligned}
$$

The extent of ordering in the equilibrium phase is characterized by the usual nematic order parameter $S$ (the orientational distribution-averaged second Legendre polynomial). In mixtures the order parameters of each of the components are defined in terms of the corresponding orientational distribution functions as

$$
S_{i}=\int P_{2}(\cos \theta) f_{i}(\theta, \varphi) d \underline{\omega},
$$

where $P_{2}(\cos \theta)=\frac{3}{2} \cos ^{2} \theta-\frac{1}{2}$. Choosing the nematic director to be oriented along the $z$ axis, the values of $S_{i}$ are between -0.5 and 1 . A negative value of $S_{i}$ corresponds to molecular ordering perpendicular to the nematic director, the isotropic orientational distribution $\left[f_{i}=1 /(4 \pi)\right]$ gives the isotropic phase value $S_{i}=0$, while a positive value of $S_{i}$ means that the ordering occurs along the nematic director. In uniaxial nematic phases the particles of one of the components are aligned along the nematic director, while those of the other component are randomly oriented in a plane perpendicular to the nematic director. In order to achieve a more efficient packing additional ordering in the perpendicular plane may take place, resulting in a biaxial order in the orientational distribution of both components. The usual nematic order parameter $S_{i}$ does not distinguish biaxial-nematic and uniaxial-nematic phases; instead a function $D(\theta, \varphi)$ $=\sin ^{2} \theta \cos (2 \varphi)$ is defined, which quantifies the ordering in the plane perpendicular to the director. In this way the biaxial-nematic order parameters are defined as

$$
\Delta_{i}=\int f_{i}(\theta, \varphi) D(\theta, \varphi) d \underline{\omega} .
$$

A value of $\Delta_{i}=0$ corresponds to uniaxial-nematic phase or isotropic phase, while $\Delta_{i} \neq 0$ indicates biaxial ordering.

The determination of the phase diagram implies the solution of the coexistence conditions (equality of pressures, and chemical potentials of each component in the coexisting phases). We solve these conditions numerically using a simplex method. ${ }^{19}$ The numerical technique, however, requires reasonable initial guesses for the packing fractions and com- positions of the coexisting phases. In order to speed up the process it is sometimes useful to perform an isotropicnematic bifurcation analysis, as this can provide an indication of the coexisting packing fractions. The bifurcation analysis is relatively simple, and it gives the isotropicnematic bifurcation packing fraction as a function of composition. The essence of the analysis is to find an infinitesimal perturbation of the isotropic orientational distribution functions which would also satisfy the coupled integral equations (8) and (9). There are many possible perturbations satisfying the integral equations, therefore the perturbation yielding the smallest packing fraction is considered as a bifurcation function. Kayser and Ravaché ${ }^{20}$ have shown that the second Legendre polynomial $P_{2}$ is the nematic bifurcation function in systems of rods. Although we use the $P_{2}$ function to determine the composition dependence of the bifurcation packing fraction, we do not follow their original method; instead our analysis is based on the expansion of the excluded volumes only up to second order in Legendre polynomials, i.e.,

$$
v_{i j}^{\mathrm{exc}} \approx y_{i j}+z_{i j} P_{2}(\cos \gamma),
$$

where $y_{i j}$ and $z_{i j}$ are the expansion coefficients. Substituting Eq. (14) into Eqs. (8) and (9) and using the Taylor series expansion for $\exp x$ up to second order (i.e., $\exp x \approx 1+x$ ) the bifurcation equation

$$
0=\left(z_{11}^{2}-z_{12}^{2}\right) x_{1} x_{2} \frac{\left(\beta F_{\mathrm{ex}}^{\mathrm{hs}} / N\right)^{2}}{v_{\mathrm{hs}}^{2}}+20 z_{11} \frac{\beta F_{\mathrm{ex}}^{\mathrm{hs}} / N}{v_{\mathrm{hs}}}+400
$$

is obtained (see Ref. 2 for more details). In the derivation of Eq. (15) we have also taken advantage of the equality of the like excluded volumes in the case of the symmetric mixture, hence Eq. (15) is symmetric in composition. Note that the bifurcation packing fraction $\eta_{\text {bif }}$ depends on the second order expansion coefficients $z_{i j}$ and the mole fraction $x_{i}$. The bifurcation packing fraction can be obtained analytically from Eq. (15) noticing that it is quadratic in $\left(\beta F_{\mathrm{ex}}^{\mathrm{hs}} / N\right) / v_{\mathrm{hs}}$, and that only one physical root of the packing fraction exists.

The Gibbs free energy $G$ is the natural choice to study phase stability in binary mixtures. The compositions and packing fractions of the coexisting phases can be determined from the points of common tangent in the $G x$ curves at a fixed pressure. The Gibbs free energy can be obtained from the Helmholtz free energy and pressure as

$$
g=\beta F / N+\beta P v_{\mathrm{hs}} / \eta,
$$

where the reduced Gibbs free energy is $g=\beta G / N$. The corresponding density (packing fraction) root that satisfies the chosen fixed pressure must be determined prior to the calculation of the Gibbs free energy. In the case of symmetric mixtures the Gibbs free energy is specially useful as this function is symmetric with respect to the equimolar composition $(x=1 / 2)$. Hence, the common tangent is always parallel to the $x$ axis, which means that phase coexistence between a rod-rich and a plate-rich phase can only take place if the mole fraction derivative of the Gibbs function for either component $x_{i}$ is zero, i.e., 


$$
\left(\frac{\partial g}{\partial x_{i}}\right)_{P}=0
$$

And it is clear that the compositions of the equilibrium phases are also symmetric. The evaluation of Eq. (17) appears to be complicated due to the constant pressure condition. However, the constant pressure derivative of the Gibbs function can be rewritten in a more suitable way as a con- stant volume (or density) derivative of the Helmholtz free energy

$$
\left(\frac{\partial g}{\partial x}\right)_{P}=\left(\frac{\partial \beta F / N}{\partial x}\right)_{\eta} .
$$

Using Eq. (1) and the phase coexistence condition given by Eq. (17) an expression

$$
\ln \left(\frac{x_{1}}{x_{2}}\right)+\sigma\left[f_{1}\right]-\sigma\left[f_{2}\right]+\frac{4 \eta-3 \eta^{2}}{(1-\eta)^{2}} \frac{\iint\left\{v_{11}^{\mathrm{exc}}\left(x_{1} f_{1} f_{1}-x_{2} f_{2} f_{2}\right)+\left(x_{2}-x_{1}\right) v_{12}^{\mathrm{exc}} f_{1} f_{2}\right\} d \underline{\omega}_{1} d \underline{\omega}_{2}}{4 v_{\mathrm{hs}}}=0
$$

can be derived for the demixing transition between rod-rich and plate-rich phases. In order to obtain the packing fraction of the demixing transition, the equations for the equilibrium orientational distribution functions [Eqs. (8) and (9)] and the equation defining the demixing curve [Eq. (19)] must be solved simultaneously. If two isotropic phases are involved, the orientational distribution function is $f_{i}=1 /(4 \pi)$, and the problem reduces to the solution of the isotropic limit of Eq. (19) given by

$$
\ln \left(\frac{x_{1}}{x_{2}}\right)+\frac{4 \eta-3 \eta^{2}}{(1-\eta)^{2}} \frac{\left(x_{1}-x_{2}\right)\left(y_{11}-y_{12}\right)}{4 v_{\mathrm{hs}}}=0 .
$$

In the derivation of this simple relation, Eq. (14) has also been used, where the first term is the exact isotropic (angle averaged) excluded volume. Equation (20) is quadratic in packing fraction, so that the isotropic-isotropic demixing packing fraction $\eta_{I-I}$ can be expressed explicitly. In the Onsager limit, where the rods are very long and thin or the plates are very flat, the packing fractions must be very small, and only the leading term $(4 \eta)$ of the Carnahan and Starling expression remains. This means that an even simpler analytical expression for the packing fraction of the demixing transition involving two isotropic phases can be obtained in the Onsager limit. In this case

$$
\eta_{I-I}=v_{h s} \frac{\ln \left(x_{2} / x_{1}\right)}{\left(x_{1}-x_{2}\right)\left(y_{11}-y_{12}\right)} .
$$

Having stated the necessary thermodynamic relations to calculate the different phase transitions with the ParsonsLee theory for symmetric mixtures, it is necessary to define the shape and geometrical parameters of the model molecules. As we have already pointed out, the constraint of having a symmetric phase diagram requires the volumes and the excluded volumes of the species to be the same. The simplest model for which the conditions of symmetry can be fulfilled is a binary mixture of hard spherocylinders. Sear and Mulder ${ }^{21}$ have shown that a symmetric binary mixture of hard spherocylinders can be constructed in the Onsager limit; both components are very long, but of different length, and the diameter of the longer molecule is smaller than that of the shorter molecule such that the components have the same excluded volumes. It is important to mention here that it is not possible, however, to construct a symmetric mixture of hard-spherocylinder particles away from the Onsager limit, i.e., when the end effects need to be incorporated (see the Appendix). In the case of uniaxial hard ellipsoid particles, a symmetric phase diagram has been determined by Camp et al., ${ }^{17,22}$ where one component is rodlike while the other is platelike. Following from the studies of Sear and Mulder ${ }^{21}$ and Camp et al., ${ }^{17,22}$ the hard cylinder model is intriguing, because at first sight both the symmetric rod-rod and rodplate mixtures appear to be feasible in such a system. Together with this, the fact that the excluded volume of hard cylinders is analytical is a particular advantage. ${ }^{23}$ Unfortunately, the conditions of symmetry mean that only a symmetric rod-plate mixture can exist, and rules out the case where both components are of rodlike shape (or both of platelike shape) (see the Appendix).

The excluded volumes between two hard cylinders of the same type $v_{11}^{\text {exc }}, v_{22}^{\text {exc }}$, or of different type $v_{12}^{\text {exc }}$ can be written in compact form as ${ }^{23}$

$$
v_{i j}^{\mathrm{exc}}(\gamma)=D_{1}^{3}\left\{a_{i j} \sin \gamma+b_{i j}+c_{i j}|\cos \gamma|+d_{i j} E(\sin \gamma)\right\},
$$

where $E(x)$ is the complete elliptic integral of the second kind defined as $E(x)=\int_{0}^{\pi / 2} \sqrt{\left(1-x^{2} \sin ^{2} \varphi\right)} d \varphi$. The parameters $a_{i j}, b_{i j}, c_{i j}$, and $d_{i j}$ depend on the molecular aspect ratios $\kappa_{1}=L_{1} / D_{1}, \kappa_{2}=L_{2} / D_{2}$ and the diameter ratio $d$ $=D_{2} / D_{1}$ of the two components. Component 1 has a length $L_{1}$ and diameter $D_{1}$, and component 2 has a length $L_{2}$ and diameter $D_{2}$. The values of the parameters $a_{i j}, b_{i j}, c_{i j}$, and $d_{i j}$ corresponding to symmetric mixtures are shown in Table I together with the values in the Onsager limit. The Legendre polynomial expansion coefficients $y_{i j}$ and $z_{i j}$ of the excluded volumes [see Eq. (14)] can be obtained using the orthogonal property of the Legendre polynomials, such that

$$
y_{i j}=\frac{1}{2} \int_{0}^{\pi} v_{i j}^{\text {exc }}(\gamma) \sin \gamma d \gamma
$$

and

$$
z_{i j}=\frac{5}{2} \int_{0}^{\pi} v_{i j}^{\text {exc }}(\gamma) P_{2}(\cos \gamma) \sin \gamma d \gamma .
$$

Before finishing this section, it is important to mention that, throughout this work, component 1 is chosen to be platelike 
TABLE I. Excluded volume coefficients for cylinder-cylinder molecules as given by Eq. (22). $\kappa_{1}=L_{1} / D_{1}$ corresponds to the plate aspect ratio, $\kappa_{2}$ $=L_{2} / D_{2}$ to the rod aspect ratio, and $d=D_{2} / D_{1}$ to the diameter ratio. For a given $\kappa_{1}$ equations (A5) and (A6) define the corresponding $d$ and $\kappa_{2}$. The corresponding values of the coefficients in the Onsager limit are also shown.

\begin{tabular}{lcccccccc}
\hline \hline & $a_{11}$ & $a_{12}$ & $b_{11}$ & $b_{12}$ & $c_{11}$ & $c_{12}$ & $d_{11}$ \\
\hline Exact & $\pi / 2+2 \kappa_{1}^{2}$ & $(\pi / 2) d(1+d)$ & $(\pi / 2) \kappa_{1}$ & $\left(\pi / 2 \kappa_{1}\right)$ & $(\pi / 2) \kappa_{1}$ & $\pi / 4\left(\kappa_{1} d^{2}+\kappa_{2} d\right)$ & $2 \kappa_{1}$ & $\left(\kappa_{1}+\kappa_{2} d\right) d$ \\
$\kappa_{2} \rightarrow \infty$ & $(\pi / 2)$ & 0 & 0 & 0 & 0 & $\left(\pi / 4 \kappa_{2} d\right)$ & 0 & 0 \\
$\kappa_{1} \rightarrow 0$ & & & & & \\
$d \rightarrow 0$ & & & & & \\
\hline \hline
\end{tabular}

$\left(\kappa_{1}<1\right)$, while component 2 is rodlike $\left(\kappa_{2}>1\right)$. In this way the diameter of the platelike component is used to define the dimensionless packing fraction and excluded volumes [see Eq. (22)].

\section{RESULTS}

In this work we have studied the phase diagrams of symmetric binary rod-plate mixtures in which the molecules are modelled as hard cylinders of different lengths and diameters. Since the aspect ratios of the rods and plates and the diameter ratio are, however, not independent, fixing the aspect ratio of the rod $\kappa_{2}$, determines the corresponding diameter ratio $d$ and aspect ratio of the plate $\kappa_{1}$ [Eqs. (A5) and (A6)]. We take into account isotropic, uniaxial-nematic, and biaxial-nematic phases, using the Parsons-Lee approach presented in the preceding section; smectic, columnar, and solid phases are not treated in this approach. Isotropicnematic phase coexistence is obtained by solving the phase boundary conditions, i.e., equality of pressure [defined by Eq. (10)] and chemical potentials [defined by Eq. (11)] in both phases. This calculation is rather intensive, as a knowledge of the equilibrium orientational distribution functions [obtained from the iterative solution of Eqs. (8) and (9)] is needed. The orientational distribution functions are also required in the calculation of the nematic-nematic demixing curves, but in these cases only Eq. (19) (instead of the equalities of pressure and chemical potentials) needs to be solved. The determination of the isotropic-isotropic coexistence is carried out straightforwardly through Eq. (20), or using the simple expression of the low-density limit [Eq. (21)]. The stability of the biaxial-nematic phase is analyzed by comparing the Gibbs free energy [Eq. (16)] of the uniaxial and biaxial nematic phases, and the bifurcation point of the two phases is defined by the point at which the two curves meet.

Since only isotropic and nematic (uniaxial or biaxial) phases are taken into account in the theory, we have restricted our study in terms of the aspect ratios and the densities considered in order to avoid systems which may undergo direct isotropic-smectic or isotropic-columnar phase transitions. In this way, we proceed from moderate rod aspect ratios $\left(\kappa_{2}=5\right)$ to the limit of very long $\left(\kappa_{2}=1066\right)$ rod aspect ratios. In Fig. 1 we present the phase diagram for a symmetric binary mixture of rods of aspect ratio of $\kappa_{2}=5$, with corresponding plates of aspect ratio $\kappa_{1} \cong 0.1571$; the diameter ratio is $d \cong 0.3155$. It is surprising to note that the a)

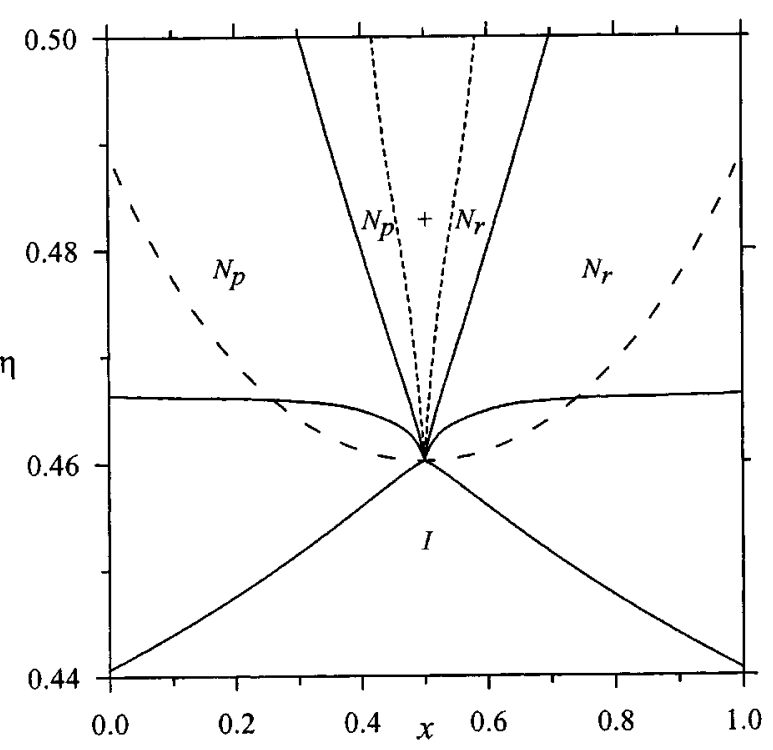

b)

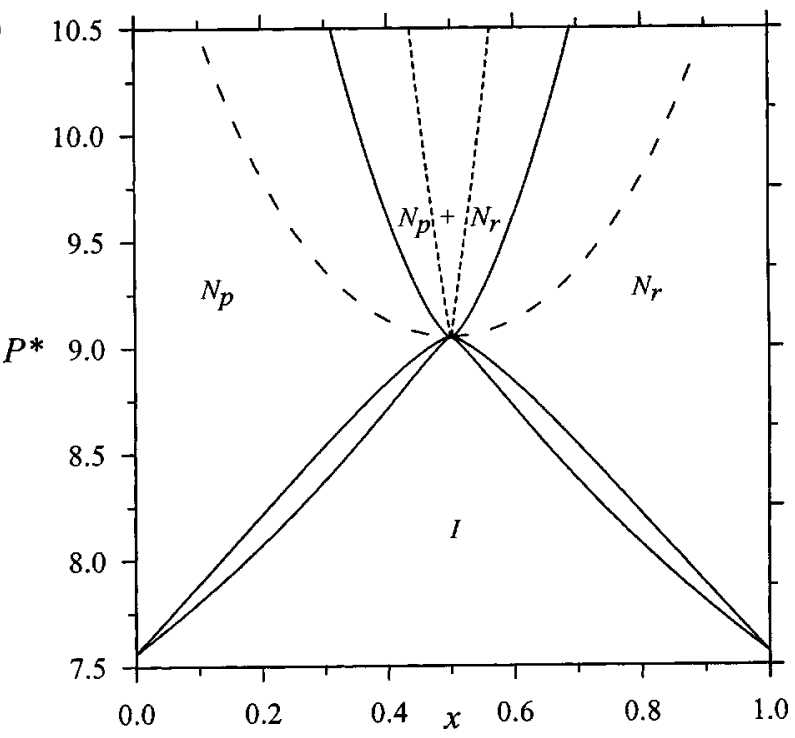

FIG. 1. Phase diagram of the symmetric rod-plate binary mixture for a system with rod aspect ratio $\kappa_{2}=5\left(\kappa_{1} \cong 0.1571, d \cong 0.3155\right)$, in (a) packing fraction composition $\eta x$, and (b) reduced pressure composition $P^{*} x$ representations. The continuous curves represent the stable coexistence curves of the isotropic-nematic and nematic-nematic demixing transitions. The dashed curves indicate the metastable nematic-biaxial nematic second-order transition. The long-dashed curve represents the isotropic-nematic bifurcation packing fractions and pressures as obtained from Eq. (15). The labels $I$, $N_{r}$, and $N_{p}$ denote isotropic, rod-rich nematic, and plate-rich nematic phases, respectively, and the mole fraction $x$ corresponds to the mole fraction of the rods. 
isotropic-nematic bifurcation packing fraction obtained from Eq. (15) significantly overestimates the range of stability of the isotropic phase; in the Onsager theory of pure hard rods ${ }^{20}$ the bifurcation density is midway between the densities of coexisting isotropic and nematic phases. Furthermore, the bifurcation analysis predicts the stabilization of the nematic phase on mixing, while the phase behavior indicates the opposite tendency (i.e., the stabilization of the isotropic phase). The isotropic-nematic transition is obtained as markedly first order for the pure components; it becomes weakly first order on mixing, and at the equimolar composition ( $x$ $=0.5$ ) becomes continuous. In the high pressure region of the diagram nematic-nematic demixing of rod-rich and plate-rich phases is observed. The Gibbs free energy of the biaxial nematic phase was investigated in this region, and it was found to be higher than that corresponding to the demixed nematic state; the biaxial phase is hence metastable (the metastable uniaxial nematic-biaxial nematic secondorder transitions are also indicated in the figure for comparison). The nematic-nematic phase separation ends at a bicritical point meeting the isotropic-nematic phase transition at a composition of $x=0.5$.

Increasing shape anisotropy stabilizes the nematic phase with respect to the isotropic phase as can be seen in Fig. 2; the phase diagram for a mixture with $\kappa_{2}=10, \kappa_{1} \cong 0.0785$, and $d \cong 0.1988$ is shown. The isotropic-nematic transition is, as before, first order in density except at $x=0.5$. Note, however, that the coexisting compositions of the isotropic and nematic phase are very close; isotropic-nematic azeotropy in the rod-rich and plate-rich regions of the phase diagram is not observed in this case, but the existence of this behavior is likely for slightly smaller aspect ratios (see Ref. 1). The transition densities are smaller than those of the previous system as a result of the higher anisotropy of the particles. Both the nematic and the isotropic coexistence packing fractions shift downwards on mixing, although no stabilization or destabilization of the phases is seen in terms of the pressure [see Fig. 2(b)]. The predicted transition densities given by the bifurcation analysis are significantly better than in the previous system, but the transition pressure is still overestimated close to the pure fluid limits. As before a stable nematicnematic demixing transition is seen in the high pressure region of the phase diagram, while the uniaxial nematicbiaxial nematic transitions are metastable. As will become clear, this is a common finding in all of the mixtures investigated in this work. The result is rather surprising as in previous work $^{1}$ the stabilization of biaxial phases was predicted for mixtures of long and flat particles. This is not the result of the scaling approach of Parsons and Lee; it turns out that excluding the end effects of the excluded volumes results in a favoring of biaxial ordering over nematic demixing. We will consider later the aspect ratios at which the end effects become negligible, and will use the Onsager theory to obtain the phase diagram of the mixture; the biaxial-nematic phase also turns out to be metastable in such a case.

Before discussing these limits further, however, it is useful to investigate the phase diagrams of mixtures with particles of increasing anisotropy. In the case of a system with $\kappa_{2}=20, \kappa_{1} \cong 0.0393$, and $d \cong 0.1252$ (Fig. 3) a pronounced a)

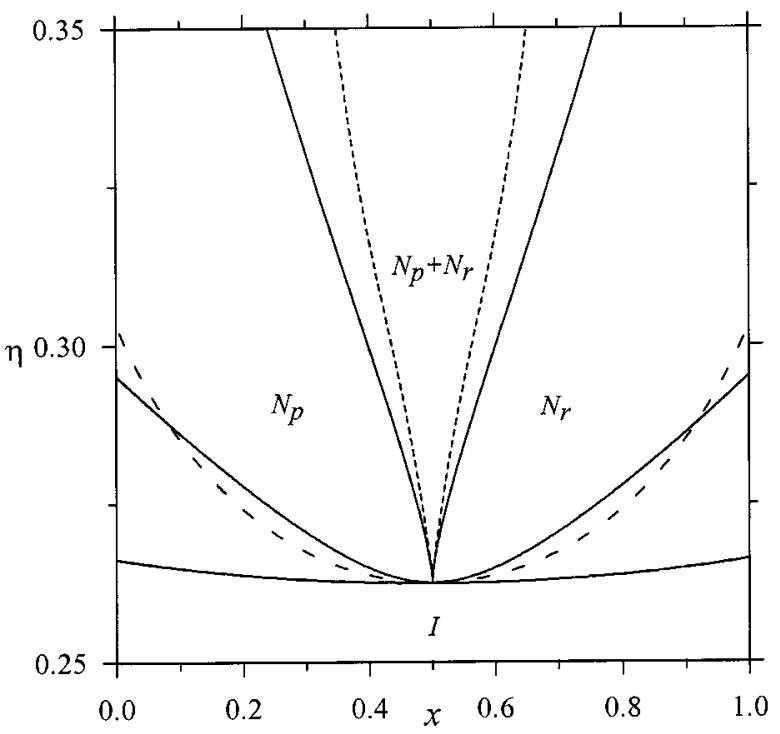

b)

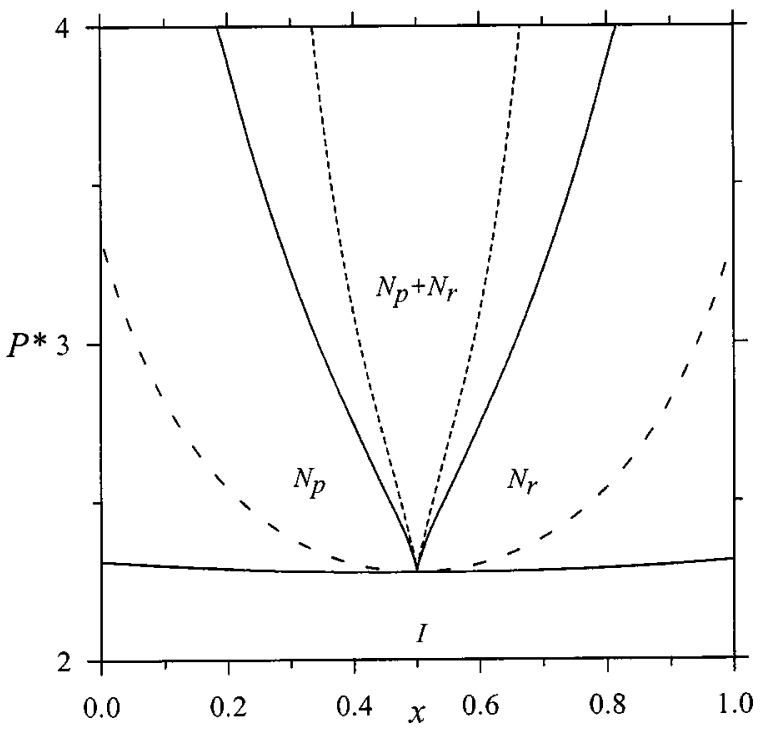

FIG. 2. Phase diagram of the symmetric rod-plate binary mixture for a system with rod aspect ratio $\kappa_{2}=10\left(\kappa_{1} \cong 0.0785, d \cong 0.1988\right)$. See Fig. 1 for details of the notation.

stabilization of the nematic phase is seen on mixing, both in terms of the packing fractions and of the pressures. The fractionation between the coexisting isotropic and nematic phases is clearly seen. In Fig. 4 the phase diagram for a rod-plate symmetric mixture with $\kappa_{2}=50, \kappa_{1} \cong 0.0157$, and $d \cong 0.0680$ is shown. There is no qualitative difference from the behavior depicted in Figs. 2 and 3 for the systems with a smaller aspect ratios. However, an interesting phenomenon is observed in the region close to the bicritical point; in the composition region of $0.4<x<0.6$ both the packing fractions and the mole fractions of the two coexisting isotropic and nematic phases are very similar, i.e., the isotropicnematic transition can essentially be considered as a secondorder transition in this composition range. This is caused by the increase in the rod-plate excluded volume in the isotropic phase as a result of the increased particle anisotropy. In a previous study ${ }^{1}$ we have shown the existence of an 
a)

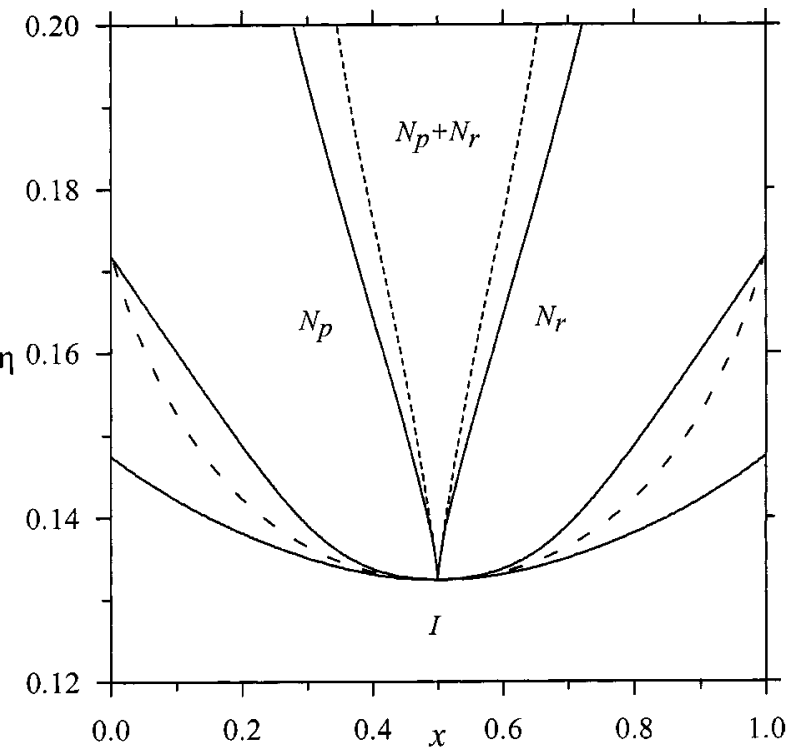

b)

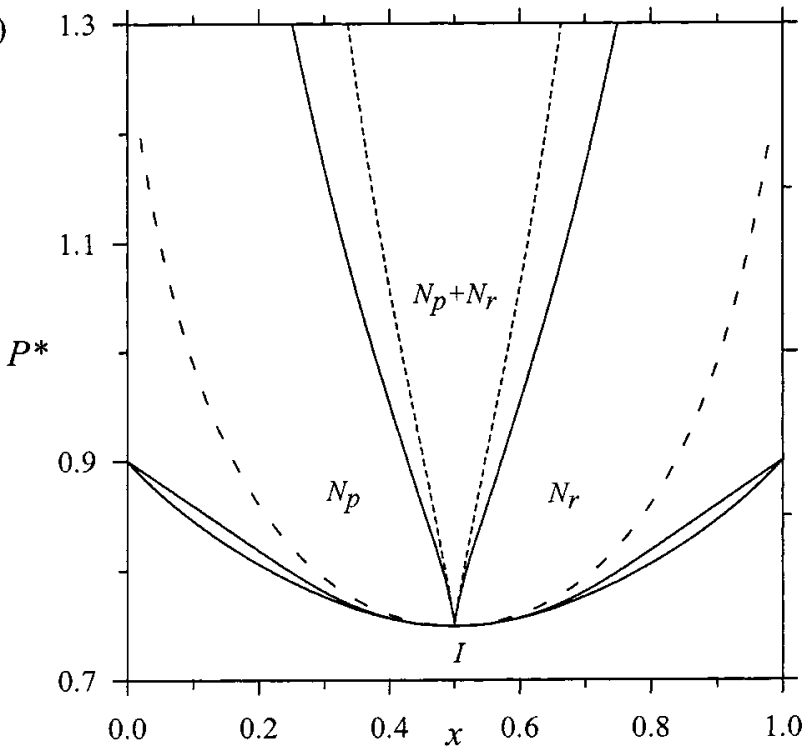

FIG. 3. Phase diagram of the symmetric rod-plate binary mixture for a system with rod aspect ratio $\kappa_{2}=20\left(\kappa_{1} \cong 0.0393, d \cong 0.1252\right)$. See Fig. 1 for details of the notation.

isotropic-isotropic demixing spinodal line in rod-plate systems; the minimum density and pressure of this spinodal instability was found to shift downwards rapidly for increasing shape anisotropy of the rods and plates. It is therefore reasonable to suggest that the spinodal instability in the isotropic phases causes the unexpected phase behavior close to the bicritical point in Fig. 4. In fact, using the spinodal ${ }^{2}$ and the bifurcation analyses presented in Sec. II, it can be shown that the spinodal decomposition curve meets the isotropicnematic bifurcation curve for a symmetric mixture with aspect ratios of $\kappa_{2}=65.5, \kappa_{1} \cong 0.0120$, and $d \cong 0.0568$ in both the pressure-mole fraction and density-mole fraction planes. This meeting point is, of course, the minimum of both functions, and takes place at the equimolar composition because of the symmetry of the functions. As a consequence, sym- a)
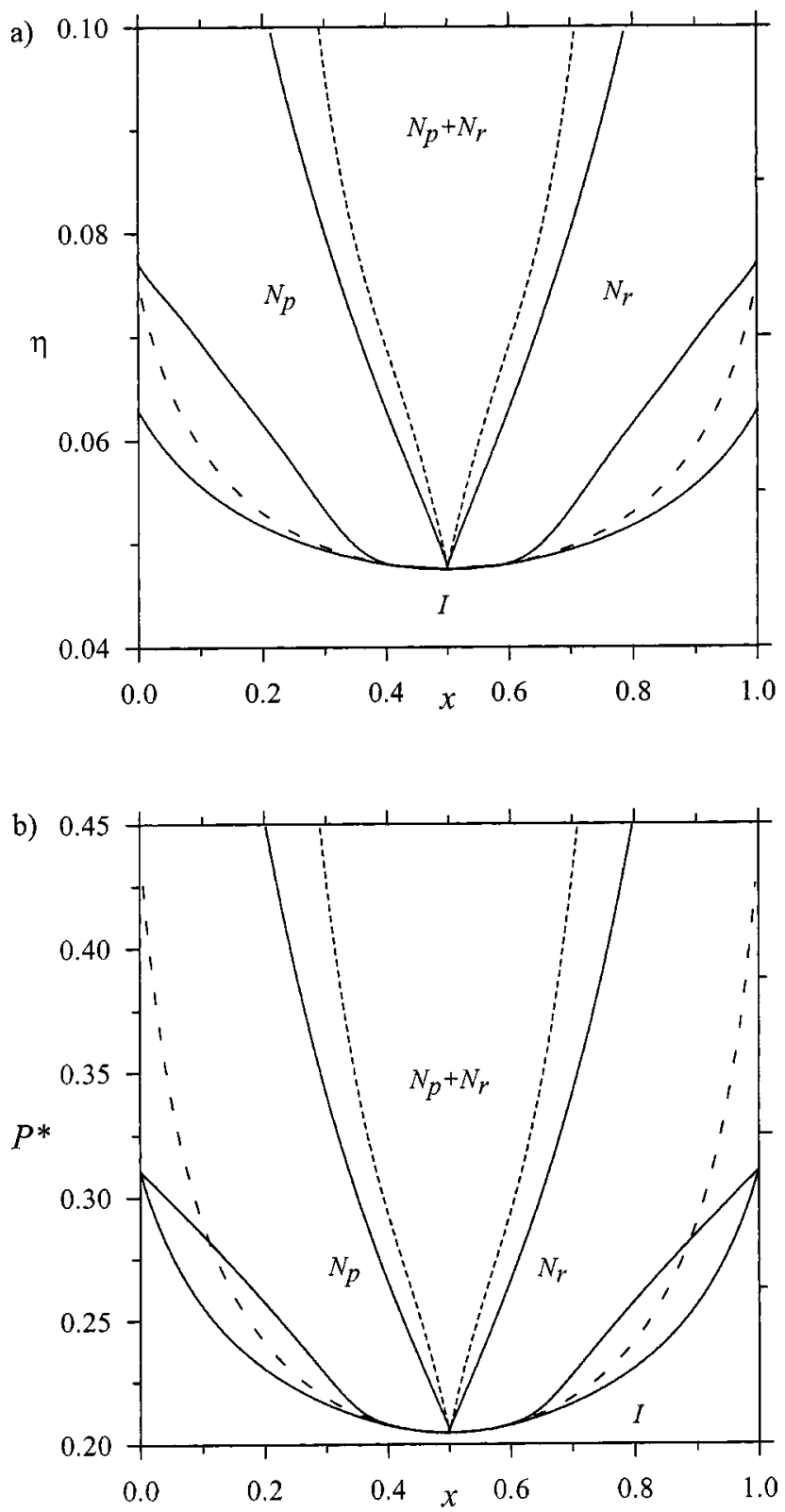

FIG. 4. Phase diagram of the symmetric rod-plate binary mixture for a system with rod aspect ratio $\kappa_{2}=50\left(\kappa_{1} \cong 0.0157, d \cong 0.0680\right)$. See Fig. 1 for details of the notation.

metric rod-plate mixtures with $\kappa_{2}>65.5$ must exhibit isotropic-isotropic demixing.

In Fig. 5 the phase diagram corresponding to a mixture with $\kappa_{2}=80, \kappa_{1} \cong 0.0098, d \cong 0.0497$ is shown. A region of isotropic-isotropic coexistence, which meets the region of nematic-nematic demixing at a four-phase point (a fourphase region in the density plane), is clearly seen. A more marked phase separation can be seen in Fig. 6. An essentially pure fluid of rods is in coexistence with one of pure plates over a wide range of pressures, and the isotropic-nematic transition is restricted to a very narrow range in pressure. The phase diagram corresponds to that of a mixture with $\kappa_{2}=200, \kappa_{1} \cong 0.0039$, and $d \cong 0.0270$. These large aspect ratios are usually considered to be well within the regime in which the Onsager limit is satisfied (i.e., in which the end effects of the excluded volumes are negligible). ${ }^{14}$ We will 
a)

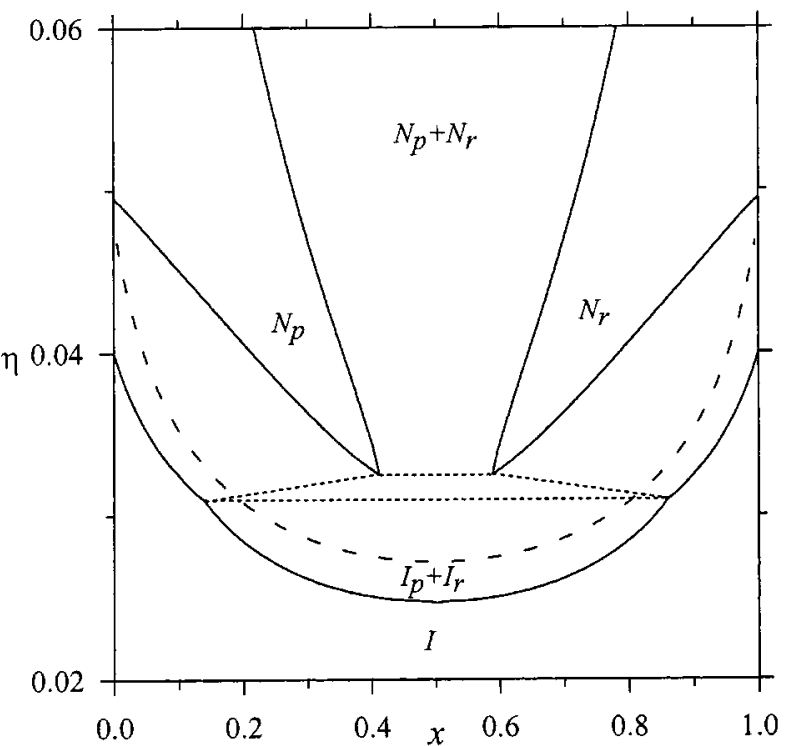

b)

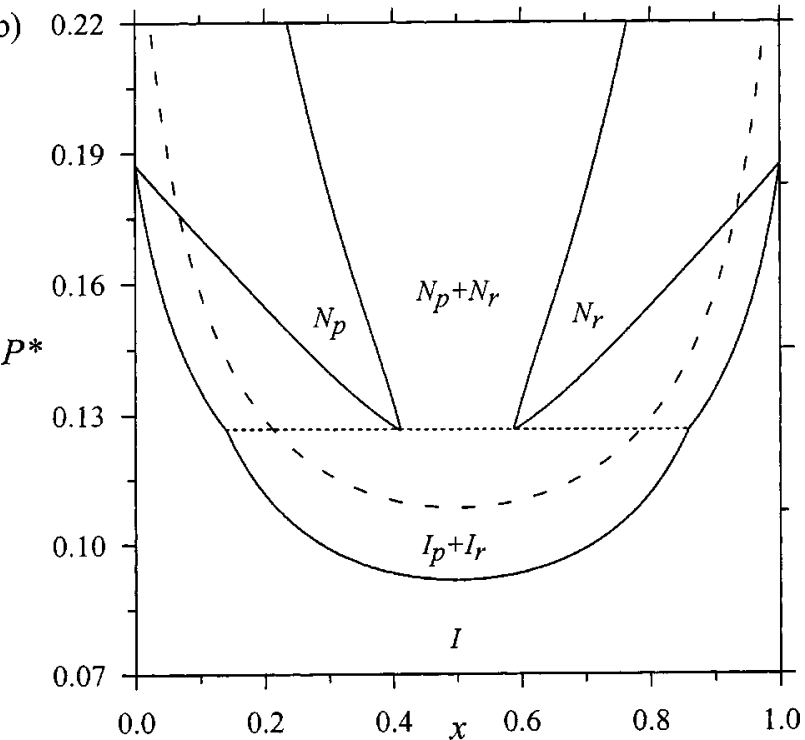

FIG. 5. Phase diagram of the symmetric rod-plate binary mixture for a system with rod aspect ratio $\kappa_{2}=80\left(\kappa_{1} \cong 0.0098, d \cong 0.0497\right)$. A region of coexistence between a rod-rich $\left(I_{r}\right)$ and plate-rich $\left(I_{p}\right)$ isotropic phases is shown. See Fig. 1 for other details of the notation.

show later, however, that this is not the case; the contribution of the end effects to the total excluded volume is still considerable, even for these aspect ratios. The densities of the two coexisting isotropic phases are very small, which means that the Parsons-Lee theory reduces to the second virial theory of Onsager at the level of the free energy [i.e., Eq. (21) can be used to calculate the demixing transition], but, as we have just mentioned, the Onsager limit of the excluded volumes is not justified.

It is useful to summarize the types of phase behavior observed in this mixture in the context of a global phase diagram. The aspect ratios of the two components in the mixture are fixed in such a way that Eq. (A6) is always fulfilled $\left(\kappa_{1} \kappa_{2}=\pi / 4\right)$. In Fig. 7 the regions in which isotropic-isotropic demixing is observed for these mixtures a)

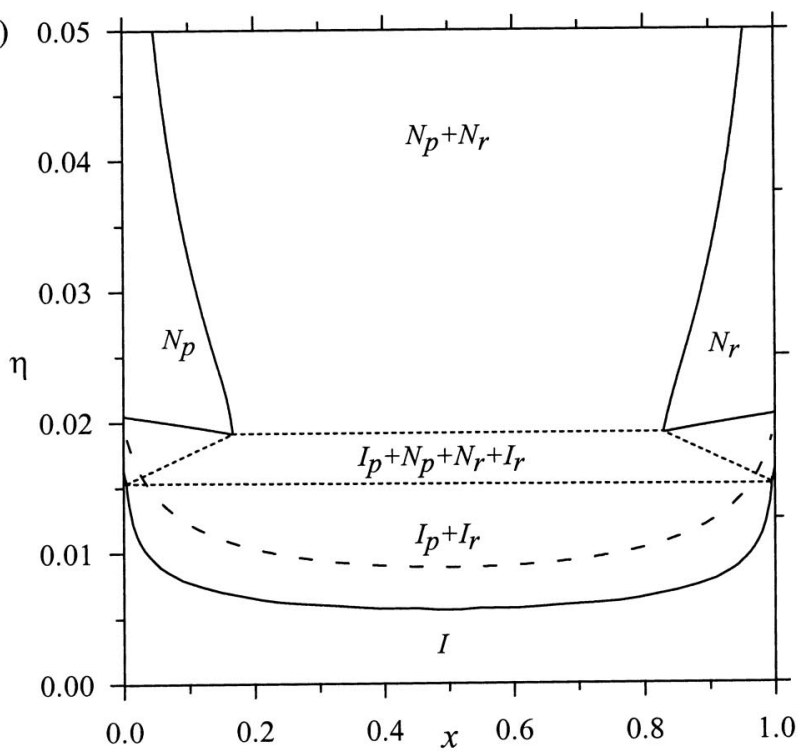

b)

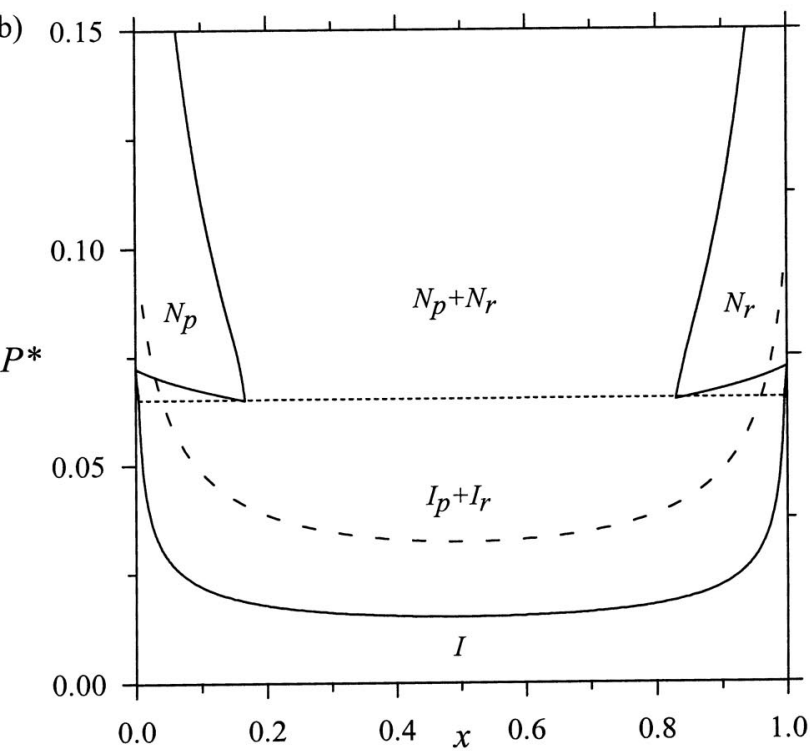

FIG. 6. Phase diagram of the symmetric rod-plate binary mixture for a system with rod aspect ratio $\kappa_{2}=200\left(\kappa_{1} \cong 0.0039, d \cong 0.0270\right)$. A region of coexistence between a rod-rich $\left(I_{r}\right)$ and plate-rich $\left(I_{p}\right)$ isotropic phases is shown. See Fig. 1 for other details of the notation.

are outlined; Eq. (A5) is satisfied along the dashed curve indicated in the figure (these are the symmetric mixtures). It should be noted that the location of the boundary curves is only approximate, because the calculation is based on a combination of spinodal demixing and isotropic-nematic bifurcation analyses (see our previous paper for details ${ }^{2}$ ). It can be seen in the figure that isotropic demixing can be observed in mixtures of particles of lower rod aspect ratios (and corresponding higher plate aspect ratio as given by the relation $\left.\kappa_{1} \kappa_{2}=\pi / 4\right)$ if the volumes of the two particles are not restricted to be equal (i.e., in nonsymmetric mixtures).

In a previous paper ${ }^{1}$ we examined the phase behavior of rod-plate mixtures using the second virial theory of Onsager, also assuming the usual Onsager limit of the excluded volumes, i.e., the terms $b_{11}, c_{11}, d_{11}, a_{12}, b_{12}$, and $d_{12}$ are 


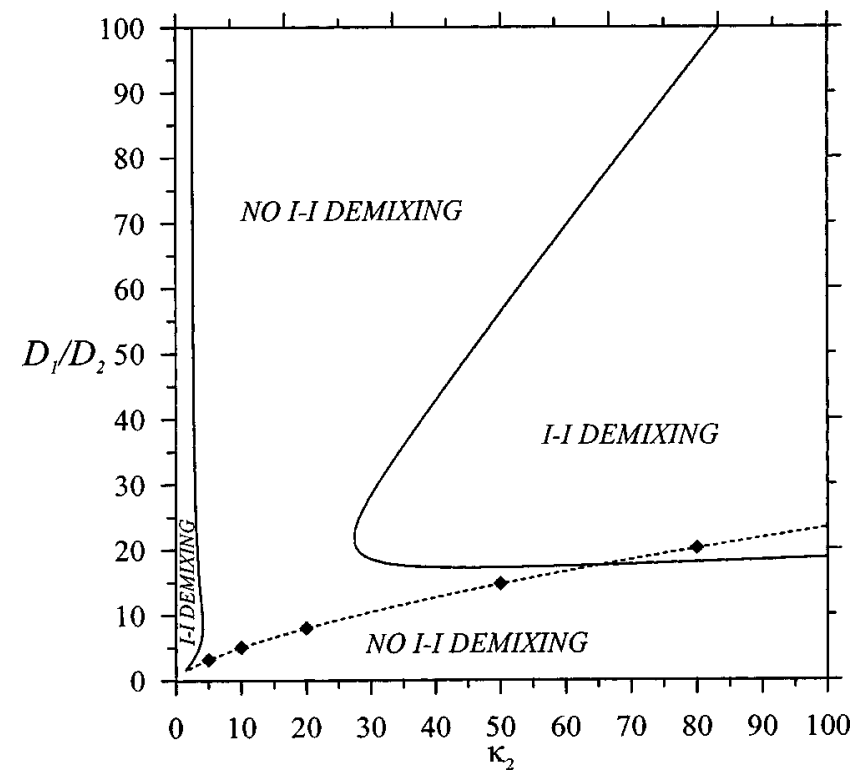

FIG. 7. Global phase diagram for mixtures of rods and plates with $\kappa_{1} \kappa_{2}$ $=\pi / 4$. The regions corresponding to molecular parameters giving rise to isotropic demixing are indicated. The dashed curve indicates the molecular parameters of the symmetric mixture of rod- and plate-like particles as given by Eqs. (A5) and (A6). The diamonds denotes the rod-plate systems studied in this work (Figs. 1-6).

taken to be zero in Eq. (22) (see Table I). The trends observed for the nematic-isotropic phase transitions (stabilization of the isotropic phase for short aspect ratios, and stabilization of the nematic phase for the longer aspect ratios) are in agreement with those found in our present work. Isotropic-isotropic separation was also observed with the Onsager theory, although for aspect ratios of the rods greater than $\kappa_{2}=133.24$, instead of the threshold value of $\kappa_{2}$ $=65.5$ found in this work. More important, however, is the fact that a biaxial-nematic phase was found to be stable for all of the molecular parameters that were studied in our previous work, ${ }^{1}$ while the nematic-biaxial nematic transition is seen to be metastable with respect to demixing into two nematic phases in all the cases studied here. Let us try to clarify this point. It is important to reiterate the fact that two separate assumptions are typically made in the so-called Onsager theories of isotropic-nematic transitions. The first is that the free energy is expanded up to the second virial coefficient only (Onsager second virial theory). We have already showed [Eqs. (20) and (21)] that if the transition densities are very low, the Parsons-Lee scaling factor reduces to the hardsphere second virial coefficient. The second approximation involves the expressions of the excluded volumes of the particles. In the so-called Onsager limit, the particles are assumed to be very long and thin rods $\left(\kappa_{2} \rightarrow \infty\right)$, and very flat and thin plates $\left(\kappa_{1} \rightarrow 0\right)$, with a vanishing diameter ratio $(d \rightarrow 0)$, so that only the leading terms of the excluded volume need to be taken into account (see Table I). In our present work we take into account the exact excluded volumes of the molecules, including the end effects, and use the Parsons-Lee expression for the free energy. It is reasonable to assume that the results of the Onsager and Parsons-Lee theories deviate for moderate aspect ratios because of the
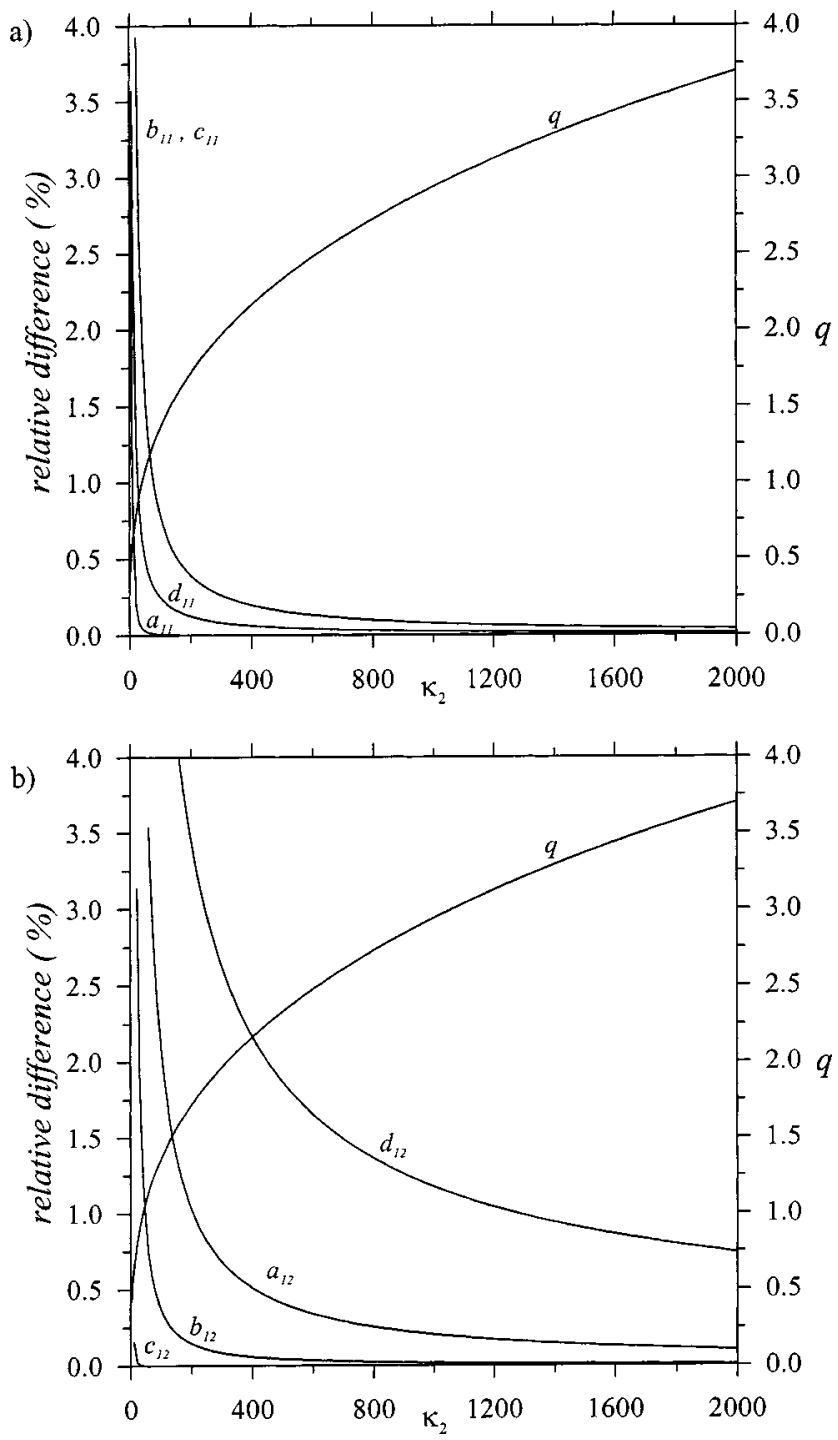

FIG. 8. Relative error in the excluded volume coefficients $a_{i j}, b_{i j}, c_{i j}, d_{i j}$, for the (a) like and (b) unlike excluded volumes tabulated in Table I [see also Eqs. (25) and (26)]. The $q$ parameter used in the Onsager theory is also depicted.

incorporation of higher virial terms (albeit approximately) in the Parsons-Lee theory. However, in the case of long rods and flat plates the two theories should become identical (we have shown that the higher virial coefficients can be neglected for aspect ratios of the order of $\kappa_{2}=200$ ). We need to consider the contribution of the end effects in the excluded volumes.

The Onsager limit of the excluded volumes corresponds to the limit of very long rods $\left(L_{2}>D_{2}\right)$, very flat plates $\left(L_{1}<D_{1}\right)$, with the diameter of the rods much smaller than that of plates $\left(D_{1}>D_{2}\right)\left(\kappa_{2} \rightarrow \infty, \kappa_{1} \rightarrow 0\right.$, and $d \rightarrow 0$ as mentioned earlier). It is useful to introduce a parameter $q\left(\kappa_{2}\right.$ $\left.=4 \pi^{2} q^{3}\right)$ which quantifies the difference between unlike and like excluded volumes in the symmetric mixture (see Ref. 1 for more details); $q=1$ corresponds to the system in which the average like and unlike virial coefficients are the same. We use this parameter, together with the relative errors of each of the contributions to the excluded volume in Fig. 8. We define the relative difference $\epsilon$ for each of the excluded 
volume terms as the ratio of the difference between the exact coefficient $\left(a_{i j}, b_{i j}, c_{i j}, d_{i j}\right)$ and the coefficients in the Onsager limit $\left(a_{i j}^{0}, b_{i j}^{0}, c_{i j}^{0}, d_{i j}^{0}\right)$, with respect to the leading term in the Onsager limit (i.e., the nonzero like, or unlike coefficient in the Onsager limit). The leading terms are the $a_{11}$ for like excluded volumes and $c_{12}$ for the unlike cases (see Table I). In this way the relative errors are given by

$$
\begin{aligned}
& \epsilon\left(a_{11}\right)=\frac{a_{11}-a_{11}^{0}}{a_{11}^{0}}, \quad \epsilon\left(b_{11}\right)=\frac{b_{11}-b_{11}^{0}}{a_{11}^{0}}, \\
& \epsilon\left(c_{11}\right)=\frac{c_{11}-c_{11}^{0}}{a_{11}^{0}}, \quad \epsilon\left(d_{11}\right)=\frac{d_{11}-d_{11}^{0}}{a_{11}^{0}},
\end{aligned}
$$

for the coefficients of the like excluded volumes, and

$$
\begin{aligned}
& \epsilon\left(a_{12}\right)=\frac{a_{12}-a_{12}^{0}}{c_{12}^{0}}, \quad \epsilon\left(b_{11}\right)=\frac{b_{12}-b_{12}^{0}}{c_{12}^{0}}, \\
& \epsilon\left(c_{11}\right)=\frac{c_{12}-c_{12}^{0}}{c_{12}^{0}}, \quad \epsilon\left(d_{11}\right)=\frac{d_{12}-d_{12}^{0}}{c_{12}^{0}},
\end{aligned}
$$

for the coefficients of the unlike excluded volumes. It can be seen from the results presented in Fig. 8(a) that the contributions of the like coefficients decay rapidly with increasing aspect ratio of the rods, so that the contribution of the lowerorder terms $\left(b_{11}, c_{11}, d_{11}\right)$ is less than $1 \%$ for aspect ratios above $\kappa_{2}=100$. This finding justifies the use of the Onsager limit of the excluded volume for pure long or flat hard-body molecules. This, however, does not turn out to be the case in mixtures. It is clear from Fig. 8(b) that the unlike excluded volume terms ( $d_{12}$ and $\left.a_{12}\right)$ make significant contributions for very large aspect ratios. Even for the largest value of the aspect ratio represented in the figure $\left(\kappa_{2}=2000\right)$, the contribution of $d_{12}$ is almost $1 \%$. The issues now are the following: Will the biaxial phases be stable for these large aspect ratios; and, Can we find equivalent systems in the Onsager (second virial free energy and leading terms of the excluded volume) and Parsons (scaled free energy and exact excluded volumes) approaches?

We have found that the two theories give qualitatively similar results for a mixture with $q=3\left(\kappa_{2}=1066\right)$. Here, the Onsager approach predicts a stable demixing transition between rodlike and platelike nematic phases instead of a stable nematic-biaxial phase. This can be seen in Fig. 9(a), where the Gibbs free energies of the nematic and biaxia nematic phases calculated with both approaches at fixed pressure (the pressure is chosen to ensure that it corresponds to states well within the nematic phase) are plotted. The Gibbs free energy obtained from the Parsons-Lee approach is higher than that of the Onsager approach [note that quantitative agreement cannot be obtained for an aspect ratio of 1066 as suggested by Fig. 8(b)], but the shape of the curves and the location of the nematic-biaxial nematic bifurcation coexistence are well represented by the Onsager approach, and the destabilization of the biaxial-nematic phase can be observed. In Fig. 9(b) the Gibbs free energy curves calculated with the Onsager approach for mixtures with $q=2$ and 2.5 $\left(\kappa_{2}=315.8\right.$ and 616.8) are shown at fixed pressure $\widetilde{P}^{*}=16$ in Onsager units $\tilde{P}^{*}=\beta P L_{2}^{2} D_{2} \pi / 4\left(\tilde{P}^{*}=\kappa_{2} P^{*}\right)$; as before,
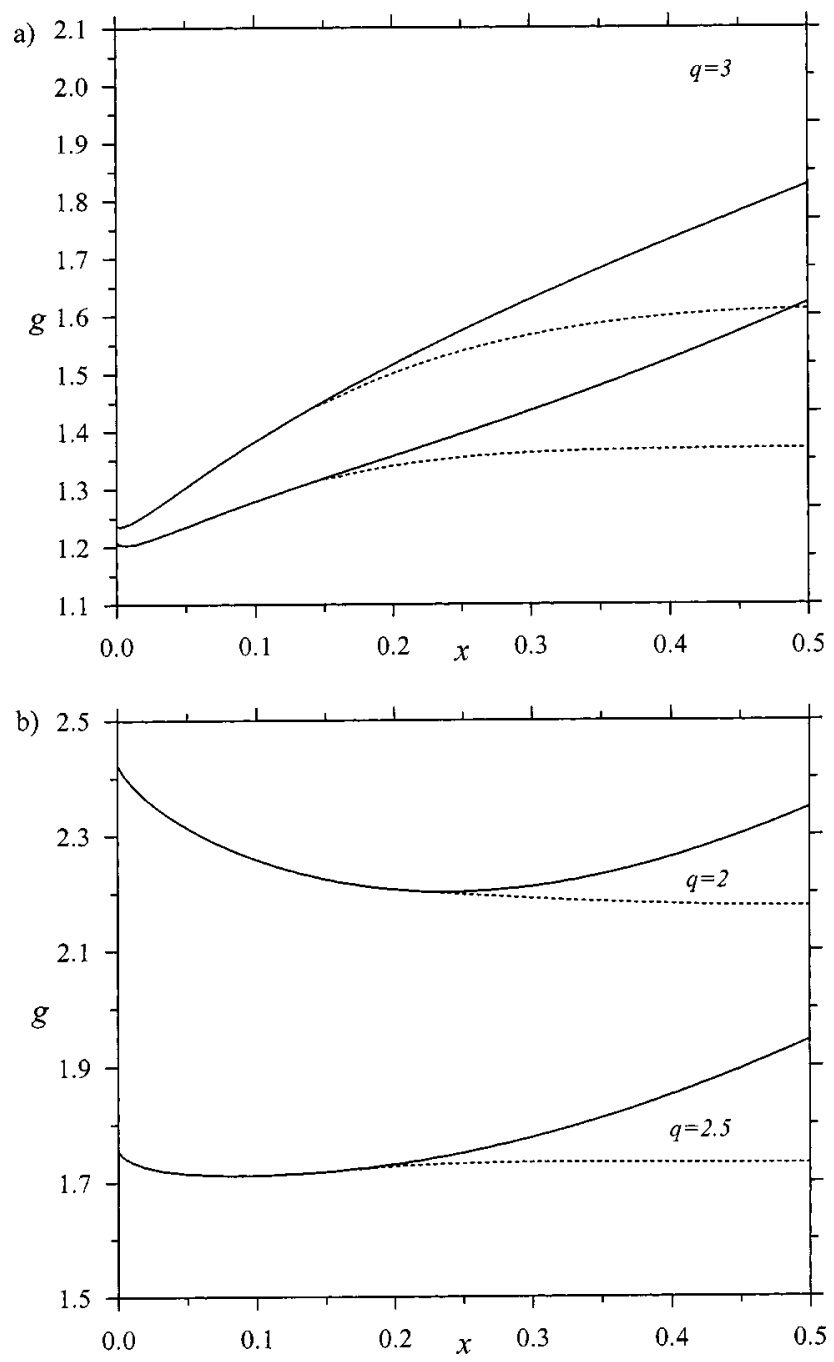

FIG. 9. (a) Gibbs free energy density $g$ versus composition of the rods $x$ at $P^{*}=0.015$ for a symmetric mixture of rods and plates obtained from Parsons-Lee approach (upper pair of curves) and the Onsager approach (lower pairs of curves) for $q=3\left(\kappa_{2}=1066\right)$. The continuous curves correspond to the plate-rich nematic phases, while the dashed curves correspond to the biaxial nematic phases. (b) The Gibbs free energy of the plate-rich nematic (continuous curve) and biaxial-nematic (dashed curve) phases are shown as obtained in the Onsager approach for $q=2\left(\kappa_{2}=315.8\right)$ and $q$ $=2.5\left(\kappa_{2}=616.8\right)$ at $P^{*}=0.051$ and 0.026 , respectively (these pressures correspond to a fixed $\widetilde{P}^{*}=16$ in Onsager units; i.e., $\left.\widetilde{P}^{*}=\beta P L_{2}^{2} D_{2} \pi / 4\right)$.

this pressure is chosen to ensure that only nematic phases are present. It can be seen that the uniaxial nematic-biaxial nematic phase transition is still stable with respect to the nematic-nematic demixing transition using the Onsager treatment with $q=2\left(\kappa_{2}=315.8\right)$. The biaxial-nematic phase is, however, found to be unstable relative to nematicnematic demixing for the system with $q=2.5\left(\kappa_{2}=616.8\right)$; this is in qualitative agreement with the Parsons-Lee theory. We can thus conclude that the Onsager approach provides a good description of the phase behavior of symmetric mixtures of rods and plates for $q>3$ (aspect ratio of the rods $\kappa_{2}=1066$ ), although it can also give an adequate qualitative description of the phase behavior for smaller values of $q$.

\section{CONCLUSION}

In this paper we have studied the phase behavior of symmetric rod-plate mixtures using the Parsons-Lee theory. 
Both components are modelled as hard cylinders. We have shown that for this molecular model, a symmetric phase diagram can only be obtained when the two components are of very different shape (i.e., rod-plate mixtures, but no rod-rod or plate-plate mixtures), and have equal molecular volume. An attempt to define a symmetric binary mixture using the hard spherocylinder model fails when the end effects are taken into account.

We have exploited the symmetry of the system and derived relatively simple equations for the isotropic-nematic bifurcation and demixing transitions (nematic-nematic as well as isotropic-isotropic demixing). The equation for the demixing transition curve is useful because the solution of phase coexistence conditions is quite demanding. Moreover, a very simple analytical equation has been derived for the isotropic-isotropic demixing packing fractions in the Onsager limit. Unfortunately, the transition properties of the isotropic-nematic phase coexistence cannot be determined from a single equation. In this case a coupled set of equations for the equality of pressure and chemical potentials is solved numerically.

As a result of the different geometry of the molecules (rod and plate) a very rich phase behavior is observed. Depending on the aspect ratio of the rodlike particles the nematic phase can be stabilized $\left(\kappa_{2}>10\right)$ or destabilized ( 5 $\left.<\kappa_{2}<10\right)$ relative to the isotropic phase. It is surprising that this tendency is not reproduced by the bifurcation analysis, which suggests that the nematic phase is stabilized relative to the isotropic phase even for very low values of $\kappa_{2}$. At high pressures nematic-nematic demixing is observed in all of the mixtures investigated. The extent of the phase separation decreases with decreasing pressure. The isotropic-nematic transition is of first order for all mole fractions except $x$ $=0.5$ where a bicritical point involving the isotropicnematic and nematic-nematic demixing transitions is observed in a number of mixtures. In the case of the mixture involving rod aspect ratios $\kappa_{2}=65.5$, an isotropic-isotropic demixing transition emerges, and the bicritical point disappears. A common feature of the phase behavior is that increasing the aspect ratio $\kappa_{2}$ enhances the fractionation between the demixed phases in both the isotropic and nematic phases.

The most surprising result is the lack of stable biaxialnematic phases in these mixtures. Using the Onsager theory (in the Onsager limit) the existence of a stable biaxialnematic phase was previously observed for all aspect ratios. ${ }^{1}$ It has been shown that the difference arises due to the fact that the lower order terms of the excluded volume, which favor the demixing transition, are not incorporated in the Onsager approach. We have shown that the Onsager limit of the excluded volumes cannot be applied below the relatively large values of the rod aspect ratio of $\kappa_{2}=1000$; this aspect ratio is orders of magnitude larger than those typically used in studies of the phase behavior with the Onsager approach.

As far as the stability of the biaxial nematic phase in uniaxial molecular system is concerned, we remark that the present theory is approximate because it is only exact at the level of the second virial coefficient, while the effect of higher-order virial terms is accounted for in an approximate manner. This approximation may be inadequate for systems with very flat platelike particles. We cannot exclude the possibility that biaxial phases might be stable in asymmetric rod-plate mixtures as a result of the subtle competition between the entropy of mixing and excluded volume entropy. However, it seems unlikely that stable biaxial-nematic phases would be observed in binary rod-plate mixtures of hard particles due to the inefficient packing of rods and plates. Biaxial phases may, however, be favored in multicomponent mixtures or polydisperse systems. ${ }^{24}$ The most promising route to stabilize the biaxial-nematic phase is probably the incorporation of attractive or hydrogen bond interactions between the unlike molecules which will increase the stability of the mixed phases. ${ }^{25,26}$ Using a meanfield theory to treat the dispersive forces, the symmetry of the binary mixture can be maintained and a simple equation can be derived for the demixing transitions to study the effect of the attractive forces on the phase behavior. This will be the subject of future work.

\section{ACKNOWLEDGMENTS}

The authors thank Demetri Photinos, Henk Lekkerkerker, Gert Vroege, and Rik Wensink for useful discussions and for sending us a preprint of their paper (see Ref. 3). S.V. would like to thank the ROPA program of the Engineering and Physical Sciences Research Council (EPSRC) for a research fellowship (GP/N03358), and A.G. would like to thank the EPSRC for the award of an Advanced Research Fellowship. The authors acknowledge support from the Joint Research Equipment Initiative (JREI) of the EPSRC for computer hardware (GR/M94427), and the Royal SocietyWolfson Foundation for the award of a refurbishment grant.

\section{APPENDIX: GEOMETRICAL CONSTRAINTS FOR SYMMETRIC MIXTURES}

In this section we derive the geometrical constraints for the molecular parameters of the particles in order to ensure that a given binary system exhibits a symmetric phase diagram, at least the level of a pair excluded volume treatment (unscaled or scaled to include the higher virial terms). Given the Onsager or Parsons approaches, the necessary and sufficient conditions for the resulting phase diagram to be symmetric are that the molecular volumes and the excluded volumes of the pure components must be equal. The geometrical requirements to satisfy these conditions obviously depend on the molecular model chosen.

Let us first consider a hard-cylinder body of length $L_{1}$, diameter $D_{1}$, and molecular volume $v_{1}=(\pi / 4) \kappa_{1} D_{1}^{3}\left(\kappa_{1}\right.$ $\left.=L_{1} / D_{1}\right)$. We can assume that the length $L_{2}$, diameter $D_{2}$ of the second component [of volume $v_{2}=(\pi / 4) \kappa_{2} D_{2}^{3}$, with $\left.\kappa_{2}=L_{2} / D_{2}\right]$ is related to those of first component by $L_{2}$ $=l L_{1}$ and $D_{2}=d D_{1}$. In this way the condition of equality of molecular volumes means that $l d^{2}=1$. The condition of equality of the like excluded volumes $\left[v_{11}(\gamma)=v_{22}(\gamma)\right]$ must be considered with care because of the angular dependence. The excluded volumes can be written as 


$$
\begin{aligned}
v_{11}(\gamma)= & D_{1}^{3}\left\{\left(\frac{\pi}{2}+2 \kappa_{1}^{2}\right) \sin \gamma+\frac{\pi}{2} \kappa_{1}\right. \\
& \left.+\frac{\pi}{2} \kappa_{1}|\cos \gamma|+2 \kappa_{1} E(\sin \gamma)\right\}
\end{aligned}
$$

and

$$
\begin{aligned}
v_{22}(\gamma)= & D_{1}^{3} d^{3}\left\{\left(\frac{\pi}{2}+2 \frac{l^{2}}{d^{2}} \kappa_{1}^{2}\right) \sin \gamma+\frac{\pi}{2} \frac{l}{d} \kappa_{1}\right. \\
& \left.+\frac{\pi}{2} \frac{l}{d} \kappa_{1}|\cos \gamma|+2 \frac{l}{d} \kappa_{1} E(\sin \gamma)\right\} .
\end{aligned}
$$

It can be shown that the equality of Eqs. (A1) and (A2) for all angles means that

$$
l=1 / d^{2}
$$

and

$$
\frac{\pi}{2}+2 \kappa_{1}^{2}=d^{3}\left(\frac{\pi}{2}+2 \frac{l^{2}}{d^{2}} \kappa_{1}^{2}\right) .
$$

The first relationship is identical to the condition of the equality of molecular volumes, while the second comes from the equality of the terms proportional to $\sin \gamma$. The solution of the coupled equations (A3) and (A4) results in a quadratic equation for the parameter $d^{3}$, so that two real solutions are obtained, one is the trivial solution ( $l=1$ and $d=1)$ and the other is nontrivial and given as

$$
d^{3}=4 \kappa_{1}^{2} / \pi \text {. }
$$

Using Eq. (A3) and the aspect ratio of component two $\kappa_{2}$, a relation

$$
\kappa_{1} \kappa_{2}=\pi / 4
$$

is found. It follows from this equation that if component one is platelike $\left(\kappa_{1}<1\right)$ the other must be rodlike $\left(\kappa_{2}>1\right)$. It is important to emphasize, hence, that is not possible to define a symmetric mixture of rod-rod or plate-plate hard cylinder molecules. It is interesting that the conditions of symmetry for phase diagrams of ellipsoidal molecules are almost the same, except that $\kappa_{1} \kappa_{2}=1$.

In binary mixtures of hard spherocylinders the excluded volumes of the two components can be written with the same scaling procedure $\left(L_{2}=l L_{1}\right.$ and $\left.D_{2}=d D_{1}\right)$ as

$$
v_{11}(\gamma)=D_{1}^{3}\left\{2 \kappa_{1}^{2} \sin \gamma+2 \pi \kappa_{1}+4 \pi / 3\right\}
$$

and

$$
v_{22}(\gamma)=D_{1}^{3} d^{3}\left\{2 \frac{l^{2}}{d^{2}} \kappa_{1}^{2} \sin \gamma+2 \pi \frac{l}{d} \kappa_{1}+4 \pi / 3\right\} .
$$

From Eqs. (A7) and (A8) two relationships

$$
l=1 / d^{2}
$$

$$
\frac{4 \pi}{3}+2 \pi \kappa_{1}=d^{3} \frac{4 \pi}{3}+2 \pi \kappa d^{2} l,
$$

are obtained. Condition (A9) is identical to (A3), but, in the case of hard spherocylinders, it does not mean that the molecular volumes of the two components are the same. Apart from the trivial solution ( $l=1$ and $d=1)$, the solution of the coupled equations (A9) and (A10) yields the condition

$$
l^{3}=\frac{-2}{3 \kappa_{1}+2} .
$$

This means that the possible values of $l$ are always unphysical, because $-1<l<0$. This indicates that symmetric phase diagrams are not possible with hard spherocylinder models, either at the level of the Parsons-Lee theory or of the Onsager theory.

${ }^{1}$ S. Varga, A. Galindo, and G. Jackson, Phys. Rev. E 66, 011707 (2002).

${ }^{2}$ S. Varga, A. Galindo, and G. Jackson, J. Chem. Phys. 117, 7207 (2002).

${ }^{3}$ H. H. Wensink, G. J. Vroege, and H. N. W. Lekkerkerker, Phys. Rev. E (to be published).

${ }^{4}$ F. M. van der Kooij and H. N. W. Lekkerkerker, Phys. Rev. Lett. 84, 781 (2000).

${ }^{5}$ F. M. van der Kooij, M. Vogel, and H. N. W. Lekkerkerker, Phys. Rev. E 62, 5397 (2000).

${ }^{6}$ F. M. van der Kooij and H. N. W. Lekkerkerker, Langmuir 16, 10144 (2000).

${ }^{7}$ F. M. van der Kooij and H. N. W. Lekkerkerker, Philos. Trans. R. Soc. London, Ser. A 359, 985 (2001).

${ }^{8}$ F. M. van der Kooij, D. van der Beek, and H. N. W. Lekkerkerker, J. Phys. Chem. 105, 1696 (2001).

${ }^{9}$ J. D. Parsons, Phys. Rev. A 19, 1225 (1979).

${ }^{10}$ S. D. Lee, J. Chem. Phys. 87, 4972 (1987).

${ }^{11}$ H. H. Wensink, G. J. Vroege, and H. N. W. Lekkerkerker, J. Chem. Phys. 115, 7319 (2001).

${ }^{12}$ S. C. McGrother, D. C. Williamson, and G. Jackson, J. Chem. Phys. 104, 6755 (1996).

${ }^{13}$ P. J. Camp, C. P. Mason, M. P. Allen, A. A. Khare, and D. A. Kofke, J. Chem. Phys. 105, 2837 (1996).

${ }^{14}$ G. J. Vroege and H. N. W. Lekkerkerker, Rep. Prog. Phys. 55, 1241 (1992).

${ }^{15}$ J. P Hansen and I. R. McDonald, Theory of Simple Liquids, 2nd ed. (Academic, London, 1986).

${ }^{16}$ J. S. Rowlinson and F. L. Swinton, Liquids and Liquid Mixtures, 3rd ed. (Butterworth Scientific, London, 1982).

${ }^{17}$ P. J. Camp and M. P. Allen, Physica A 229, 410 (1996).

${ }^{18}$ A. Galindo, S. Varga, A. J. Haslam, A. Vanakaras, and D. J. Photinos (unpublished).

${ }^{19}$ W. H. Press, B. P. Flannery, S. A. Teukolsky, and W. T. Vetterling, Numerical Recipes in Fortran, 1st ed. (Cambridge University Press, London, 1986).

${ }^{20}$ R. F. Kayser and H. J. Ravaché, Phys. Rev. A 17, 2067 (1978).

${ }^{21}$ R. P. Sear and B. M. Mulder, J. Chem. Phys. 105, 7727 (1996).

${ }^{22}$ P. J. Camp, M. P. Allen, P. G. Bolhuis, and D. Frenkel, J. Chem. Phys. 106, 9270 (1997)

${ }^{23}$ L. Onsager, Ann. N.Y. Acad. Sci. 51, 627 (1949).

${ }^{24}$ Y. Martínez-Ratón and J. A. Cuesta, Phys. Rev. Lett. (to be published).

${ }^{25}$ A. G. Vanakaras and D. J. Photinos, Mol. Cryst. Liq. Cryst. 299, 65 (1997).

${ }^{26}$ A. G. Vanakaras, S. C. McGrother, G. Jackson, and D. J. Photinos, Mol. Cryst. Liq. Cryst. 323, 199 (1998).

and 